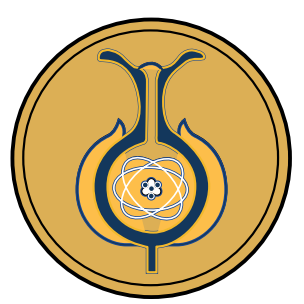

\title{
Actitud hacia la matemática de los padres y las madres de estudiantes de secundaria
}

\author{
Attitude towards mathematics from parents of secondary students
}

Atitude com relação à matemática de pais e mães de estudantes do ensino médio

\author{
Luis Gerardo Meza-Cascante ${ }^{1}$ Zuleyka Suárez-Valdés-Ayala ${ }^{1}$ Evelyn Agüero-Calvo ${ }^{1} \bullet$ \\ Martha Calderón-Ferrey ${ }^{2} \bullet$ Rodolfo Jiménez-Céspedes $^{3} \bullet$ Laura Sancho-Martínez $^{4} \bullet$ \\ Patricia Pérez-Tyteca $^{5} \bullet$ Javier Monje-Parrilla $^{5}$
}

\section{Received: Mar/26/2020 • Accepted: Aug/28/2020 • Published: Jan/31/2021}

\section{Resumen}

El objetivo principal de la investigación fue estudiar el nivel de actitud hacia la matemática de los padres y las madres de familia de estudiantes de la educación secundaria oficial diurna costarricense, analizando la existencia de diferencias por sexo, por nivel de estudio y rango de edad. La investigación es de tipo cuantitativa descriptiva-exploratoria. La muestra estuvo integrada por 1851 personas padres 0 madres de estudiantes de la educación secundaria pública, académica y diurna costarricense; el diseño muestral fue simple aleatorio estratificado, según la zona de ubicación (rural o urbana) y área geográfica (provincia) a la que pertenecía el estudiantado matriculado en el 2019. Asimismo, las técnicas estadísticas aplicadas fueron la t de Student y ANOVA de un factor para el estudio de las hipótesis de la investigación. Como resultado se infiere que existen diferencias entre hombres y mujeres en el nivel de actitud hacia la matemática, mostrando los primeros niveles superiores con tamaño del efecto bajo. Además, se detectaron diferencias entre las personas con educación primaria incompleta o educación secundaria incompleta con las que tienen educación universitaria completa, mostrando niveles mayores de actitud los últimos. También, se detectaron diferencias en la actitud hacia la matemática entre las personas con edades entre los 35 y los 50 años, con las de edades mayores, con tamaño del efecto bajo. Se concluye que un $75.6 \%$ de las personas encuestadas manifestó niveles de actitud hacia la matemática altos, lo que se valora como un hallazgo positivo.

Palabras clave: educación matemática; actitud hacia la matemática; educación secundaria; Costa Rica; padres y madres.

\footnotetext{
Luis Gerardo Meza-Cascante $\$ gemeza@tec.ac.cr, (1) https://orcid.org/0000-0002-5413-0172

Zuleyka Suárez-Valdés-Ayala \zsuarez@tec.ac.cr, (D) https://orcid.org/0000-0002-1822-4825

Evelyn Agüero-Calvo $\$ evaguero@tec.ac.cr, (D) https://orcid.org/0000-0002-2294-0357

Martha Calderón-Ferrey $₫$ micalderon@tec.ac.cr, $\mathbb{D}$ https://orcid.org/0000-0002-0571-0859

Rodolfo Jiménez-Céspedes $\triangle$ rodjimenez@tec.ac.cr, (1) https://orcid.org/0000-0002-4704-6351

Laura Sancho-Martínez \lasancho@tec.ac.cr, (1) https://orcid.org/0000-0002-0946-9505

Patricia Pérez-Tyteca \ patricia.perez@ua.es, (D) https://orcid.org/0000-0002-7796-9042

Javier Monje-Parrilla $\triangle$ monjejavier@ua.es, (D) https://orcid.org/0000-0003-0020-2991

1 Escuela de Matemática, Instituto Tecnológico de Costa Rica, Cartago, Costa Rica.

2 Escuela de Ciencias Sociales, Instituto Tecnológico de Costa Rica, Cartago, Costa Rica.

3 Escuela de Ciencias Naturales y Exactas, Instituto Tecnológico de Costa Rica, Cartago, Costa Rica.

4 Escuela de Cultura y Deporte, Instituto Tecnológico de Costa Rica, Cartago, Costa Rica.

5 Departamento de Innovación y Formación Didáctica, Universidad de Alicante, Alicante, España.
} 


\section{Abstract}

The main objective of the paper was to study the level of attitude parents of Costa Rican regular secondary school students have towards mathematics, by analyzing differences between sex, levels of education, and age ranges. This paper is a quantitative descriptive exploratory research. The sample consisted of 1851 parents of Costa Rican public, academic, and daytime secondary school students. The design was a stratified simple random sample, based on the location (rural or urban) and the geographical area (province) students were in when enrolled in 2019. In addition, the statistical techniques applied to study the research hypotheses are Student's t-distribution and one-way ANOVA. As a result, it is inferred that there are differences between men and women in their level of attitude towards mathematics, showing the first higher levels with low effect size. There were also differences between parents with incomplete primary or secondary education with those who completed college, the latter showing higher levels of attitude. Differences were also detected in the attitude towards mathematics between people ages 35-50 with those older, with low effect size. It is concluded that $75.6 \%$ of the surveyed parents showed high levels of attitude towards mathematics, which is a positive finding.

Keywords: mathematics education, attitude towards mathematics, secondary education, Costa Rica, parents

\section{Resumo}

0 principal objetivo desta pesquisa foi estudar o nível de atitude com relação à matemática de pais e mães de família de estudantes do ensino médio oficial diurno costarriquenho, analisando a existência de diferenças por sexo, por nível de estudo e faixa etária. A pesquisa foi quantitativa descritiva exploratória. A amostra constituiu de 1851 pessoas: pais ou mães de estudantes do ensino médio público, acadêmico e diurno costarriquenha, o desenho de amostragem foi simples aleatória estratificada, segundo a zona de localização (rural ou urbana) e área geográfica (distrito) a que pertencia o corpo estudantil matriculado em 2019. Da mesma forma, as técnicas estatísticas aplicadas foram o teste t de Student e a ANOVA de um fator para o estudo das hipóteses da pesquisa. Como resultado, infere-se que existem diferenças entre homens e mulheres no nível de atitude com relação à matemática, mostrando os primeiros níveis superiores com tamanho do efeito pequeno. Além disso, foram detectadas diferenças entre as pessoas com 0 ensino fundamental incompleto ou o ensino médio incompleto com as que têm o ensino superior completo, mostrando níveis mais altos de atitude dentre os últimos mencionados. Inclusive, foram detectadas diferenças na atitude com relação à matemática entre as pessoas com idades na faixa dos 35 e 50 anos, e com idades superiores, um tamanho do efeito pequeno. Conclui-se que $75,6 \%$ das pessoas respondentes manifestaram níveis de atitude com relação à matemática altos, 0 que se avalia como um resultado positivo.

Palavras-chave: educação matemática, atitude com relação à matemática, ensino médio, Costa Rica, pais e mães

\section{INTRODUCCIÓN}

El interés por estudiar la incidencia de la familia en los procesos educativos formales, en general, y en la enseñanza y el aprendizaje de la matemática, en particular, data de varios años. En una breve línea de tiempo, es posible encontrar numerosas investigaciones sobre la temática.

Un trabajo de Poffenberger y Norton (1959) señala que los comportamientos del hogar desempeñan papeles fundamentales en la determinación de las actitudes hacia la matemática. Banks (1964, citado en Quiles, 
1993, p. 116) afirmaba que "las actitudes matemáticas de los padres y profesores explican buena parte de la varianza de las actitudes de los alumnos hacia la asignatura".

Así, la participación de padres y madres, incluso de modo inconsciente, puede ejercer considerable influencia sobre la actitud de sus hijos ante la matemática (Cockcroft, 1985). Las formas de actuar de los padres y las madres —más positivas habitualmente las de los primeros (Visser, 1987) - también intervienen en el rendimiento de sus descendientes, de modo que los estudiantes con padres y madres que brindan más apoyo en sus tareas de matemáticas, además de presentar actitudes más positivas hacia la materia, obtienen un mayor rendimiento (Cai, Moyer y Wang, 1999).

Podemos observar, entonces, cómo la actitud de padres y madres es un pilar fundamental para el sustento y fortalecimiento de una actitud positiva en el estudiantado. Igualmente, esta importancia se ve reflejada en el hecho de que el interés por la temática no ha decrecido a lo largo de los años, sino que, por el contrario, se ha fortalecido, tanto en el ámbito internacional como en el nacional (costarricense).

En este sentido, Farr (2015) determinó que la mayoría de los padres, madres e hijos entrevistados reportó actitudes positivas hacia la matemática. Además, la indagación sugiere que "tipos indirectos de involucramiento de los padres y madres, como sus actitudes hacia la matemática, pueden ser más influyentes en la actitud de los niños hacia la matemática que los tipos directos de participación como el acompañamiento en la resolución de tareas" (p. 251).

Cervantes, Arenas y Aroca (2016) mostraron que la actitud de los padres de familia afecta el proceso de formación de sus hijos en matemática; incide de modo positivo o negativo en el proceso de aprendizaje de ellos, aunque en este trabajo no se comprueba con cuánta intensidad sucede.

En una línea similar, Knap, Landers y Liang (2016) sostienen que las actitudes tanto de los padres como de las madres hacia la matemática y su confianza para aprender esta disciplina impactan el rendimiento académico del estudiantado en matemática.

Mohr-Schroeder et al. (2017) encontraron correlaciones positivas y estadísticamente significativas entre la actitud de los padres y de las madres hacia la matemática y la de sus hijos. De la misma manera, hallaron que "la actitud de los padres y madres funge como un factor de predicción relevante de la actitud de sus hijos hacia la matemática" (p. 220).

Haque y Farhana (2017) hallaron que el comportamiento de los padres y de las madres tiene un efecto significativo sobre la ansiedad matemática de su progenie, tanto que "el $17 \%$ de la ansiedad matemática de los estudiantes de secundaria se debe a la actitud de los padres y madres hacia la matemática". Además, descubrieron que "el género de los padres tiene un efecto significativo sobre la ansiedad matemática de los hijos, mientras que el género de los hijos no tiene un efecto significativo sobre su ansiedad matemática" (p. 13).

Los resultados de Soni y Kumari (2015) sugieren que la ansiedad matemática y la postura de los padres hacia esta materia "actúan como precursoras de la ansiedad matemática y de la actitud matemática de sus hijos" (p. 344), inclusive, intervienen significativamente sobre el rendimiento académico matemático de estos.

Por otra parte, Davadas y Lay (2018) concluyen que variables como el apoyo positivo de los profesores y la instrucción en el aula predicen la actitud de los 
estudiantes hacia la matemática, en mayor medida que la influencia de la actitud de los padres y las madres.

En el ámbito costarricense, Sanabria (2016) estudió la relación entre el constructo "actitud hacia la matemática", la "percepción de la actitud de la madre hacia la matemática" y la "percepción de la actitud del padre hacia la matemática" en la educación media costarricense. Encontró relaciones significativas (a un nivel de significancia del 0.01) y positivas, entre ellas: moderada $(\mathrm{r}=0.312)$ entre la primera y la segunda, débil $(r=0.262)$ entre la primera y la tercera y moderada $(r=0.666)$ entre las dos últimas. Además, notó que la "percepción de la actitud de la madre hacia la matemática" aporta más explicación de la varianza de la variable "actitud hacia la matemática" $(\beta=$ 0.248) que la "percepción de la actitud del padre hacia la matemática” ( $\beta=0.097)$.

Leonhardes (2017) desarrolló un trabajo sobre la relación entre las variables "percepción de la actitud de la madre hacia la matemática", "percepción de la actitud del padre hacia la matemática" y "expectativa de éxito en matemática" de los estudiantes de tres colegios públicos de la provincia de Cartago, Costa Rica. Halló correlación positiva y moderada entre las dos primeras variables $(\mathrm{r}=0.628, \mathrm{p}=0<0.01)$, la primera y la tercera $(\mathrm{r}=0.459, \mathrm{p}=0<0.01)$ y la segunda $\mathrm{y}$ la tercera $(\mathrm{r}=0.424, \mathrm{p}=0<0.01)$.

Por otra parte, los resultados de Meza et al. (2019) muestran relación significativa, positiva y moderada, entre las variables "actitud hacia la matemática" y "percepción de la actitud del padre hacia la matemática" ( $\mathrm{r}=0.567, \mathrm{p}=0<0.01)$; relación significativa, positiva y moderada, entre las variables "actitud hacia la matemática" y "percepción de la actitud hacia la matemática del padre" $(\mathrm{r}=0.494, \mathrm{p}=0<0.01)$, y relación significativa, positiva y moderada, entre las variables "percepción de la actitud hacia la matemática de la madre" y "percepción de la actitud hacia la matemática del padre" $(\mathrm{r}=0.605, \mathrm{p}=0<0.01)$.

La síntesis de los resultados que se reseñan en investigaciones realizadas, tanto en el nivel internacional como nacional, develan la importancia de elementos del espectro afectivo, como la actitud o las creencias de los padres y de las madres, en el comportamiento hacia la matemática de sus descendientes. Por tanto, la trascendencia de continuar con el estudio de las actitudes hacia la matemática, en particular de los padres y de las madres, se justifica porque las indagaciones van mostrando que el papel desempeñado en la dimensión afectiva de los hijos, respecto a esta materia, puede ser determinante en su proceso de enseñanza-aprendizaje.

La búsqueda de estudios similares llevados a cabo en Costa Rica, en bases de datos (Scopus, Web of Science, Redalyc, Scielo, EBSCO y Dialnet), en repositorios institucionales (Kimuk, RepositorioTEC), metabuscadores (BASE y OpenDoar) y catálogos de universidades costarricenses (Universidad de Costa Rica, Instituto Tecnológico de Costa Rica, Universidad Nacional y Universidad Estatal a Distancia), con los términos de búsqueda mathematics, attitude, father, mother, parents, family, education, attitude toward mathematics y Costa Rica, así como en su versión en español, no arrojó ningún resultado que coincida con la temática principal de la investigación reportada en este artículo, a saber, la actitud hacia la matemática de los padres y la madres de estudiantes de educación media. Por esta razón, los resultados de este trabajo se consideran pioneros en el ámbito costarricense. 


\section{METODOLOGÍA}

Este estudio tiene carácter descriptivo-exploratorio y un enfoque, eminentemente, cuantitativo. La caracterización de las personas participantes en la investigación, la identificación del instrumento de medición aplicado, el procedimiento para la recolección de los datos y la descripción de las técnicas estadísticas utilizadas se presentan en esta sección.

\section{Participantes}

En este trabajo, fueron seleccionados padres y madres de estudiantes matriculados en colegios diurnos y oficiales costarricenses en el 2019. El muestreo se efectuó en etapas: en primer lugar, se seleccionaron colegios por muestreo simple aleatorio estratificado, según la zona de ubicación (rural o urbana) y provincia de Costa Rica; en segunda instancia, se eligieron estudiantes de las segundas secciones de cada nivel educativo (en el colegio que fue elegido). Luego se le asignó a cada estudiante un formulario por hogar el cual fue completado por las personas encargadas, padre o madre, y una vez completado fue devuelto por el estudiantado al colegio. De esta manera, se conformó una muestra con 1851 personas, la distribución que se presenta en la tabla 1 .

\section{Variables}

Las variables consideradas en el estudio, más su definición conceptual y operativa, se presentan en la tabla 2.

\section{Instrumento de medición}

La actitud de los padres y las madres hacia la matemática fue medida por medio del instrumento de "Actitudes matemáticas de los padres", elaborada por Quiles (1993). Está integrada por 23 reactivos que se miden por medio de una escala tipo Likert; concretamente, esta última ofrece cinco opciones, desde "totalmente de acuerdo" a "totalmente en desacuerdo", y mostró un valor de alfa de Cronbach de 0.89 en el estudio original (Quiles, 1993).

La validez del instrumento se estudió a partir de los datos recabados para la investigación, cuyos resultados se reportan en este trabajo, mediante el cálculo del índice

Tabla 1. Distribución de la muestra obtenida en el estudio, según condición parental, edad $y$ nivel educativo, mes, año

\begin{tabular}{llll}
\hline \multicolumn{1}{c}{ Variable de estudio } & \multicolumn{1}{c}{ Categorías de respuesta } & Frecuencia & Porcentaje \\
\hline Tipo de relación parental & Madre & 1225 & 66.2 \\
& Padre & 626 & 33.8 \\
Edad en rangos & Menores de 35 & 346 & 18.7 \\
& Entre 35 y 50 & 1193 & 64.45 \\
Nivel educativo repor- & Mayores de 50 & 312 & 16.85 \\
tado & Primaria incompleta & 266 & 14.4 \\
& Primaria completa & 414 & 22.4 \\
& Secundaria incompleta & 426 & 23.0 \\
& Secundaria completa & 270 & 14.6 \\
& Universitaria incompleta & 207 & 11.2 \\
& Universitaria completa & 268 & 14.5 \\
\hline
\end{tabular}

Nota: Fuente propia de la investigación. 
Tabla 2. Definición conceptual y operativa de las variables en el estudio

\begin{tabular}{|c|c|c|}
\hline Variable & Definición conceptual & Definición operativa \\
\hline Condición parental & $\begin{array}{l}\text { Se refiere a la relación de la persona que } \\
\text { responde el formulario: papá o mamá. }\end{array}$ & $\begin{array}{l}\text { Según indicación de la persona } \\
\text { informante en el formulario } \\
\text { aplicado. }\end{array}$ \\
\hline Nivel de estudio & $\begin{array}{l}\text { Corresponde al nivel de educación más alto } \\
\text { que la persona ha concluido. }\end{array}$ & $\begin{array}{l}\text { Primaria incompleta, primaria } \\
\text { completa, secundaria incomple- } \\
\text { ta, secundaria completa, superior } \\
\text { incompleta o superior completa. }\end{array}$ \\
\hline Rango de edad & $\begin{array}{l}\text { Rango de edad del informante: menor de } 35 \\
\text { años, de } 35 \text { a } 50 \text { y mayor de } 50 \text { años. }\end{array}$ & $\begin{array}{l}\text { Según indicación de la persona } \\
\text { informante en el formulario } \\
\text { aplicado. }\end{array}$ \\
\hline Actitud hacia la matemática & $\begin{array}{l}\text { Valoración positiva o negativa hacia la } \\
\text { matemática. Reactivos de } 1 \text { a } 23 \text { de la escala } \\
\text { "Actitudes matemáticas de los padres" } \\
\text { Quiles (1993). }\end{array}$ & $\begin{array}{l}\text { Puntaje obtenido en la escala } \\
\text { "Actitudes matemáticas de los } \\
\text { padres" Quiles (1993). }\end{array}$ \\
\hline
\end{tabular}

Nota: Fuente propia de la investigación.

de discriminación de cada ítem, realizando el cálculo de la correlación entre la puntuación aportada por el ítem y la obtenida por la suma de los otros ítems (restando el ítem evaluado) e interpretando su valor de acuerdo con la baremación recomendada en Lozano y De La Fuente-Solana (2013). Todos los ítems superaron el valor mínimo de 0.3 establecido en esa baremación.

Se determinó, además, el supuesto de unidimensionalidad de la escala, aplicando el análisis factorial, por ser la técnica más utilizada para estos efectos (Jiménez y Montero, 2013) para evidenciar que mide esencialmente un solo constructo, calculando, de previo, el índice de adecuación muestral de Kaiser-Meyer y Olkin (KMO), con un valor de 0.96 y de esfericidad de Bartlett $(\mathrm{p}=0$ $<0.05$ ). Como criterio de unidimensionalidad, se usó el recomendado en Carmines y Zeller (1979, citado en Burga, 2006), el cual requiere que el primer factor explique al menos el $40 \%$ de la varianza (42.99\% para este estudio).

La confiabilidad (o fiabilidad de la escala) se examinó con la técnica alfa de Cronbach, con un valor de 0.938 , lo que indica, siguiendo a Cea (1999), que posee una confiabilidad adecuada.

\section{Procedimiento}

El instrumento se aplicó a los padres y las madres del segundo grupo de cada nivel en cada colegio seleccionado, siguiendo el procedimiento aplicado en Quiles (1993), consistente en enviar los formularios a los hogares, con los estudiantes. Estos eran los encargados de solicitar a sus parientes que completaran la herramienta y de regresarla a la institución para ser recopilada por un docente colaborador (responsable de hacerla llegar al grupo de investigación).

\section{Análisis estadístico}

El análisis estadístico descriptivo de los datos considera una baremación de los puntajes para establecer una distribución en dos categorías: baja y alta, según el puntaje fuera menor o mayor, respectivamente, al valor medio de la escala.

Posteriormente, con la finalidad de inferir resultados, se contrastaron las siguientes hipótesis: 
- $\quad$ Hipótesis 1: No existen diferencias en la actitud hacia la matemática, entre padres y madres.

- Hipótesis 2: No existen diferencias en la actitud hacia la matemática, según el nivel de estudio.

- Hipótesis 3: No existen diferencias en la actitud hacia la matemática, según el rango de edad.

Para el caso de la primera hipótesis, se aplica la prueba paramétrica t de Student, asumiendo la normalidad de la distribución de los datos, con base en el teorema del límite central, considerando que cada una de las categorías, en contraste, tiene más de 100 elementos. Para las hipótesis restantes, se empleó la prueba ANOVA, cuando la prueba de Levene detectó homogeneidad de varianzas y la de Welch lo contrario, todas complementadas con la prueba, a posteriori, de Scheffé. En todos los casos, hubo una significancia del $5 \%$.

Para los casos con diferencias estadísticamente significativas, se calcula el tamaño del efecto, empleando la $d$ de Cohen (Morales-Vallejo, 2012), la cual estima el número de desviaciones típicas que separan a dos grupos. La interpretación de la magnitud de los tamaños del efecto medidos por la $d$ de Cohen se realizó a partir de las referencias dadas por Cohen (Morales-Vallejo, 2012): en torno a 0.20 (diferencia pequeña), 0.50 (diferencia moderada) y 0.80 o más (diferencia grande).

\section{ANÁLISIS Y RESULTADOS}

\section{Clasificación del nivel de actitud hacia la matemática}

El nivel medio de actitud hacia la matemática $(\mathrm{M}=81.89, \mathrm{SD}=18.78)$ fue significativamente mayor que el promedio de la escala $(\mathrm{t}(1850)=29.542, \mathrm{p}=0<0.05)$, lo que indica que, globalmente, los padres y madres de la muestra presentan un nivel de actitud hacia la matemática superior al promedio; es decir, manifiestan, en general, niveles positivos de actitud hacia la matemática.

La clasificación de los puntajes obtenidos para el comportamiento hacia la matemática indica $75.6 \%$ para la categoría de actitud positiva y $24.4 \%$ para la de actitud negativa.

\section{Contraste de la hipótesis 1}

Para el contraste de la primera hipótesis, se comparó la media de los padres $(\mathrm{M}=$ $85.38, \mathrm{SD}=20.44)$ con la media de las madres $(\mathrm{M}=80.11, \mathrm{SD}=17.61)$ y se encontró una diferencia estadísticamente significativa $(\mathrm{t}(1849)=5.500, \mathrm{p}=0<0.05)$. Lo anterior quiere decir que se rechaza la hipótesis nula y se acoge la alternativa, la cual indica que existen diferencias en el nivel de actitud hacia la matemática entre padres y madres, con media superior para los hombres y un tamaño del efecto $(d=0.2834)$ interpretado como una distinción pequeña.

\section{Contraste de la hipótesis 2}

Para el análisis de la segunda hipótesis, se comparó la media de las seis categorías establecidas, utilizando la prueba de Welch. En este sentido, la prueba de Levene $(p=0.03<0.05)$ rechazó la hipótesis de igualdad de varianzas; así mismo, se determinó que existen diferencias, estadísticamente, significativas entre al menos un par de medias de los cinco niveles ( $F$ $(5,772.735)=5.489, \mathrm{p}=0<0.05)$.

De este modo, se utilizó la prueba post hoc de Scheffé, para comparar los diferentes pares de medias y se demostró que la de actitud hacia la matemática es significativamente menor entre las personas con primaria incompleta $\left(\mathrm{M}_{\mathrm{pi}}=78.34, \mathrm{SD}_{\mathrm{pi}}=16.32, \mathrm{p}<\right.$ 
0.05) y las que tienen educación universitaria completa $\left(\mathrm{M}_{\mathrm{uc}}=83.54, \mathrm{SD}_{\mathrm{uc}}=21.43, \mathrm{p}<\right.$ 0.05), además, el tamaño del efecto fue bajo $(d=0,2728)$. Así mismo, el otro caso correspondió a personas con formación secundaria incompleta $\left(\mathrm{M}_{\mathrm{si}}=80.28, \mathrm{SD}_{\mathrm{si}}=18.46, \mathrm{p}\right.$ $<0.05)$ y aquellas que tienen la universitaria completa $\left(\mathrm{M}_{\mathrm{uc}}=83.54, \mathrm{SD}_{\mathrm{uc}}=21.43, \mathrm{p}<\right.$ $0.05)$, igual que el caso anterior, dejan ver un tamaño del efecto bajo $(\mathrm{d}=0.1658)$.

En otras palabras, existen diferencias estadísticamente significativas en el nivel de actitud hacia la matemática entre los padres y las madres que tienen educación primaria incompleta o secundaria incompleta, comparados con los que tienen formación universitaria completa, con mayor valor medio para los últimos.

\section{Contraste de la hipótesis 3}

Para el análisis de la tercera hipótesis, se comparó la media de las tres categorías establecidas, utilizando la prueba de ANOVA, dado que la de Levene $(p=0.569$ $>0.05)$ mostró la existencia de igualdad de varianzas. Usando la prueba post hoc de Scheffé, se encontró que la media de "actitud hacia la matemática" es significativamente menor para las personas del rango de edad entre 30 y 50 años $(\mathrm{M}=81.33$, $\mathrm{SD}=17.91, p=0.025<0.05) \mathrm{y}$ las mayores de $50(\mathrm{M}=84.57, \mathrm{SD}=18.93, p=$ $0.025<0.05)$, con tamaño de efecto bajo $(d=0.178)$, sin que se detectaran diferencias estadísticamente significativas en el nivel de actitud hacia la matemática entre los otros rangos de edad.

\section{Discusión de los resultados}

La investigación estudió la actitud hacia la matemática de los padres y las madres del estudiantado de colegios académicos, oficiales y diurnos, matriculados en el 2019 en Costa Rica.

Los resultados indican que, aproximadamente, $75.6 \%$ de las personas participantes en la investigación mostró una actitud positiva hacia la matemática, en función del instrumento utilizado, lo que se estima como un hallazgo favorable; se confirma con el hecho de que el valor medio de la variable en la muestra es significativamente mayor que la media de la escala. Este descubrimiento resulta, de alguna manera, sorprendente, porque existe evidencia de que la matemática puede ser percibida como difícil, aburrida, confusa, frustrante, estresante, poco práctica, compleja y abstracta (Gil, Guerrero y Blanco, 2006; Meza y Azofeifa, 2011). Desde una perspectiva práctica, dicha averiguación implica que, en la tarea de motivar el aprecio por el estudio de la matemática, especialmente en el estudiantado, se puede contar con el elemento positivo de que sus padres y madres muestren una actitud favorable hacia la disciplina, porque, tal como han evidenciado Cervantes, Arenas y Aroca (2016), la actitud de los progenitores puede incidir en el proceso de aprendizaje de sus hijos.

Las diferencias en el nivel de comportamiento hacia la matemática, identificadas entre padres y madres, con actitudes más positivas en los hombres, son concordantes con los hallazgos de otras investigaciones realizadas en Costa Rica, en el dominio afectivo, en el campo de la matemática, en los que las mujeres han mostrado resultados menos favorables (Meza, Suárez y Agüero, 2015; Agüero, Calderón, Meza y Suárez, 2016; Agüero et al., 2017; Agüero, Meza y Suárez, 2017; Delgado, Espinoza y Fonseca, 2017; Meza, Agüero y Suárez, 2019).

La investigación no aporta información sobre las causas que puedan explicar 
las diferencias, dado su carácter descriptivo. Algunos autores (Perina, 2002; Reyes, 1984) han sugerido que tales divergencias podrían deberse a que las mujeres estén más dispuestas a responder de manera más sincera que los hombres en las temáticas tratadas, pero nada en este estudio parece dar conclusión sobre eso.

Para fines prácticos, el encontrar las disimilitudes mencionadas, al tener un tamaño del efecto bajo, implica que en los emprendimientos desarrollados para mantener o mejorar la actitud de los padres y las madres no parece ser necesario enfocar, de manera diferenciada, a los hombres y a las mujeres.

Las distinciones detectadas en el nivel de conducta hacia la matemática entre las personas con primaria incompleta o secundaria incompleta, en comparación con las que tienen educación universitaria completa, con actitud mayor para las últimas, presentan tamaños bajos del efecto, lo que conlleva concluir que pueden tener poca importancia práctica.

La indagación tampoco aporta datos sobre las razones que puedan aclarar las diferencias en el nivel de actitud hacia la matemática entre los rangos de nivel de estudios indicados. En una línea especulativa, con la intención de visualizar posibles explicaciones e identificar futuras agendas de investigación, parece observarse que las personas con educación universitaria completa, al tener una formación académica de mayor profundidad, han experimentado diversas situaciones que les llevan a tener una actitud más positiva de la matemática, mientras que los de primaria incompleta y los de secundaria incompleta pueden, por el contrario, haber enfrentado circunstancias que les resultaran frustrantes y que, incluso, podrían ser parte de las razones por las que no tuvieron oportunidad de concluir sus estudios, aquellas que les llevan a tener una actitud menos positiva hacia la matemática.

No obstante, a partir de lo hallado, conviene valorar si en los procesos enfocados en mejorar la conducta hacia la matemática cabe diferenciar entre quienes tienen educación primaria o secundaria incompleta y los que han logrado formación universitaria completa, con la finalidad de mejorar la eficacia de esas intervenciones.

El estudio detectó desemejanzas en el nivel de actitud hacia la matemática entre las personas cuyo rango de edad es 35-50 años y las de edades mayores, con niveles, en promedio, más bajos para las primeras. Aunque la investigación no aporta datos sobre las razones que justifiquen la diferencia, se sugiere, para futuros trabajos, indagar si puede deberse a que la mayor experiencia acumulada a lo largo de vivencias personales y laborales contribuye a que las personas aprecien, de mejor manera, la utilidad y la importancia de la matemática, lo que podría incidir en esa actitud mejorada hacia la disciplina.

\section{CONCLUSIONES}

La investigación permite concluir que el nivel medio de actitud hacia la matemática fue significativamente mayor que el promedio de la escala, lo que indica, globalmente, sobre las personas en estudio, que presentan un nivel de comportamiento hacia la matemática superior al promedio; es decir, manifiestan, en general, niveles altos de conducta hacia la matemática.

La clasificación de los puntajes obtenidos para la actitud hacia la matemática indica que un $75.6 \%$ de personas manifestó comportamientos positivos y un 24.4 $\%$, negativos. Se encontraron distinciones, en las personas investigadas, en el nivel de conducta hacia la matemática entre padres 
y madres, con media superior para los hombres y con un tamaño del efecto que se interpreta como diferencia pequeña.

Se detectaron diferencias estadísticamente significativas en el nivel de actitud hacia la matemática entre los padres y las madres: aquellos que tienen educación primaria incompleta o educación secundaria incompleta, comparados con los que poseen formación universitaria completa, con mayor valor medio para los últimos y tamaño pequeño de los efectos.

Finalmente, se hallaron diferencias en el nivel de actitud hacia la matemática según rangos de edad: entre las personas de 35-50 años y las de edades mayores, con una conducta, en promedio, más favorable para las últimas.

\section{RECONOCIMIENTOS}

Trabajo desarrollado en el marco del proyecto de investigación "AMAPA: Estudio de la actitud hacia la matemática de los padres y madres de familia de estudiantes de la educación secundaria costarricense", bajo el código 0144-0038. Se agradece el apoyo de la Vicerrectoría de Investigación y Extensión del Instituto Tecnológico de Costa Rica.

\section{DECLARACIÓN DE LA CONTRIBUCIÓN DE LOS AUTORES}

El porcentaje total de contribución para la conceptualización, preparación y corrección de este artículo fue el siguiente: L. G. M. $25 \%$, Z. S. V-A. $25 \%$, E. A. C. 25 $\%$, M. C. F. $5 \%$, L. S. M. $5 \%$, R. J. C. $5 \%$, P. P-T. $5 \%$, J. M. P. $5 \%$.

\section{DECLARACIÓN DE DISPO- NIBILIDAD DE LOS DATOS}

Los datos que respaldan los resultados de este estudio serán puestos a disposición por el autor correspondiente [L. G. M], previa solicitud razonable.

\section{REFERENCIAS}

Agüero, E.; Calderón, M.; Meza, L. G. y Suárez, Z. (2016). Relación entre autoestima y autoconfianza matemática en estudiantes de educación media costarricense. Revista Comunicación, 25(2), 4-13. https://doi.org/10.18845/ rc.v25i2-16.3302

Agüero, E.; Meza, L. G. y Suárez, Z. (2017). Attitude toward usefulness of mathematics of Costa Rican high school students. Modern Journal of Language Teaching Methods (MJLTM), 7(2), 162-168. Recuperado de https://mjltm. org/article-1-71-en.html

Agüero, E.; Meza, L. G.; Suárez, Z. y Schmidt, S. (2017). Estudio de la ansiedad matemática en la educación media costarricense. Revista Electrónica de Investigación Educativa, 19(1), 35-45. https://doi.org/10.24320/ redie.2017.19.1.849

Burga, A. (2006). La unidimensionalidad de un instrumento de medición: Perspectiva factorial. Revista de Psicología, 24(1), 53-80. Recuperado de https://www.redalyc.org/ pdf/3378/337829536003.pdf

Cai, J.; Moyer, J. y Wang, N. (1999). Parental Roles in Students' Learning of Mathematics: An Exploratory Study. Research in Middle Level Education Quarterly, 22(3), 1-18. Recuperado de https://www.tandfonline.com/doi/abs/1 0.1080/10848959.1999.11670147

Cea, M. A. (1999). Metodología cuantitativa: estrategias y técnicas de investigación social. Madrid: Síntesis.

Cervantes, J.; Arenas, J. y Aroca, A. (2016). Actitudes recíprocas entre padres de familia e hijos en el proceso de formación matemática. Revista educación y desarrollo social, 10(1), 98-111. Recuperado de https://revistas.unimilitar.edu.co/index.php/reds/article/view/1451 
Cockcroft, W. H. (1985). Las matemáticas sí cuentan. Madrid: MEC.

Davadas, S. D. y Lay, Y. F. (2018). Factors Affecting Students' Attitude toward Mathematics: A Structural Equation Modeling Approach. Eurasia Journal of Mathematics, Science and Technology Education, 14(1), 517-529. https://doi.org/10.12973/ejmste/80356

Delgado, I.; Espinoza, J. y Fonseca, J. (2017). Ansiedad matemática en estudiantes universitarios de Costa Rica y su relación con el rendimiento académico y variables sociodemográficas. Propósitos y Representaciones, 5(1), 275-324. http://dx.doi.org/10.20511/ pyr2017.v5n1.148

Farr, S. M. (2015). The Role of Parents in Children's Attitudes towards Mathematics. (Thesis, Master of Education). University of Waikato, Hamilton, New Zealand. Recuperado de https://hdl.handle.net/10289/10007

Gil, N.; Guerrero, E. y Blanco, L. (2006). El dominio afectivo en el aprendizaje de las matemáticas. Revista Electrónica de Investigación Psicoeducativa, 4(1), 47-72. Recuperado de https:// www.redalyc.org/pdf/2931/293123488003.pdf

Haque, M. y Farhana, K. (2017). Relationship between Parent's Attitude towards Math and Children's Math Anxiety. Journal of Child and Adolescent Behavior, 5(4), 11-13. http:// dx.doi.org/10.4172/2375-4494.1000354

Jiménez, K. y Montero, E. (2013). Aplicación del modelo de Rasch, en el análisis psicométrico de una prueba de diagnóstico en matemática. Revista digital Matemática, Educación e Internet, 13(1), 1-23. Recuperado de https:// tecdigital.tec.ac.cr/revistamatematica/ARTICULOS_V13_N1_2012/RevistaDigital Montero_V13_n1_2012/Screen_RevistaDigital_Montero_V1 $\overline{3}$ n1_2012.pdf

Knap, A.; Landers, R. y Liang, S. (2016). How does parental attitude toward mathematics prompt student achievement? En M. B. Wood, E. E. Turner, M. Civil y J.A. Eli (eds.), Proceedings of the 38th annual meeting of the North American Chapter of the International Group for the Psychology of Mathematics Education (pp. 1107-1110).

Leonhardes, E. (2017). Estudio sobre "percepción de la actitud de la madre hacia la matemática" y "percepción de la actitud del padre hacia la matemática" y su relación con "la expectativa de éxito en matemática" de las y los estudiantes de tres colegios públicos de la provincia de Cartago. (Tesis de licenciatura no publicada). Instituto Tecnológico de Costa Rica, Costa Rica.

Lozano, L. y De la Fuente-Solana, E. (2013). Diseño y validación de cuestionarios. En A. Pantoja-Vallejo (ed.), Manual básico para la realización de tesinas, tesis y trabajos de investigación (pp. 251-274).

Meza, L. G. y Azofeifa, R. (2011). Actitud hacia la matemática de las y los estudiantes de undécimo año de los colegios del Cantón Central de Cartago. En Memorias del VII Congreso Internacional de Enseñanza de la Matemática asistida por Computadora (VII CIEMAC). Cartago, Costa Rica.

Meza-Cascante, L. G.; Agüero-Calvo, E. y Suárez-Valdés-Ayala, Z. (2019). Mathematical Self-Confidence of High School Students: A Study in Costa Rica. Revista Electrónica Educare, 23(1), 1-15. https://doi. org/10.15359/ree.23-1.3

Meza-Cascante, L. G.; Agüero-Calvo, E.; Suárez-Valdés-Ayala， Z.; Calderón-Ferrey, M.; Sancho-Martínez, L.; Pérez-Tyteca, P. y Monje-Parrilla. J. (2019). Actitud hacia la matemática: percepción de la actitud de padres. Revista Comunicación, 28(1), 4-15. https://doi.org/10.18845/rc.v28i1-2019.4437

Meza-Cascante, L. G.; Suárez-Valdés-Ayala, Z. y Agüero-Calvo, E. (2015). Resolución de problemas matemáticos en la educación media costarricense: un estudio acerca de la actitud. Revista Comunicación, 24(2), 58-69. https:// doi.org/10.18845/rc.v24i2.2487

Mohr-Schroeder, M.; Cavalcanti, M.; Schroeder, C.; Jackson, C.; Jong, C. y Speler, L. (2017). Parents' Attitudes Toward Mathematics and the Influence on Their Students' Attitudes toward Mathematics: A Quantitative Study. School science and mathematics, 117(5), 214-222. https://doi.org/10.1111/ssm.12225

Morales-Vallejo, P. (2012). El tamaño del efecto (effect size): análisis complementarios al contraste de medias. Recuperado de https:// web.upcomillas.es/personal/peter/investigacion/Tama\%floDelEfecto.pdf

Perina, K. (2002). Sum of all fears. Psychology Today. Recuperado de https://www. psychologytoday.com/us/articles/200211/ sum-all-fears 
Poffenberger, T. y Norton, D. (1959). Factors in the formation of attitudes toward mathematics. The Journal of Educational Research, 52(5), 171-176. https://doi.org/10.1080/00220671.1 959.10882562

Quiles, M. N. (1993). Actitudes matemáticas y rendimiento escolar. Comunicación, Lenguaje y Educación, 5(18), 115-125. https://doi.org/10 $.1080 / 02147033.1993 .10821078$

Reyes, L. H. (1984). Affective variables and mathematics education. The Elementary School Journal, 84(5), 558-581. https://doi. org/10.1086/461384

Sanabria, J. (2016). Estudio de los niveles de "actitud hacia la matemática", "percepción de la actitud del padre hacia la matemática” y "percepción de la actitud de la madre hacia la matemática” que manifiestan los estudiantes de los colegios Francisca Carrasco Jiménez, San Luis Gonzaga y Experimental Bilingüe José Figueres Ferrer en el año 2015. (Tesis de licenciatura no publicada). Instituto Tecnológico de Costa Rica, Costa Rica.

Soni, A. y Kumari, S. (2015). The Role of Parental Math Anxiety and Math Attitude in Their Children's Math Achievement. International Journal of Science and Mathematics Education, 15(2), 331-347. https://doi.org/10.1007/ s10763-015-9687-5

Visser, D. (1987). The Relationship of Parental Attitudes and Expectations to Children's Mathematics Achievement Behaviour. The Journal of Early Adolescence, 7(1), 1-12. https://doi. org/10.1177/0272431687071002

\section{(c) $\underset{B Y}{(\rightarrow)}=$}

Actitud hacia la matemática de los padres y las madres de estudiantes de secundaria (Luis Gerardo Meza-Cascante • Zuleyka Suárez-Valdés-Ayala • Evelyn Agüero-Calvo • Martha Calderón-Ferrey • Rodolfo Jiménez-Céspedes • Laura Sancho-Martínez • Patricia Pérez-Tyteca • Javier Monje-Parrilla) Uniciencia is protected by Attribution-NonCommercial-NoDerivs 3.0 Unported (CC BY-NC-ND 3.0) 